\title{
Imaging with ESCA of Paper Containing a Polyacrylamide Dry Strength Resin
}

\author{
Takushi Sakaemura $^{* 1,2}$, Fumiaki Inokuchi ${ }^{* 1}$, and Tatsuo Yamauchi ${ }^{* 2}$ \\ ${ }^{* 1}$ R\&D Department, Arakawa Chemical Industries, Ltd., 1-1-9, Tsurumi, Tsurumi-ku, Osaka, \\ Osaka 538-0053, Japan \\ ${ }^{* 2}$ Graduate School of Agriculture, Kyoto University, Sakyo-ku, Kyoto 606-8502, Japan
}

\begin{abstract}
In order to examine the distribution of a polyacrylamide (PAM) on exact fiber surface, the latest imaging with a scanning ESCA microprobe, which analytical depth is up to $10 \mathrm{~nm}$, was adapted to papers containing a cationic PAM dry strength resin. The exact surface PAM contents were significantly higher than the total PAM content, which indicates that the PAM added would be concentrated nearby the surface of fibers. And the degree of the concentration decreased with increasing in beating degree. $\mathrm{C} 1 \mathrm{~s}$ and $\mathrm{O} 1 \mathrm{~s}$ images apparently showed unevenness due to the surface roughness of paper irrespective of beating degree of the fibers, although these images derived from cellulosic fibers may be expected to show their uniform distributions. On the other hand N1s image derived from the PAM gave isolated and scattered signals. This characteristic distribution of the PAM on fiber surface differed from the generally uniform in-plane distribution of the PAM within a fiber wall as shown in the imaging with a microscopic ATR/FT-IR in which the analytical depth is about $1 \mu \mathrm{m}$.
\end{abstract}

(Received 10 October, 2009; Accepted 6 January, 2010)

\section{Introduction}

The present paper making frequently employs various types of chemical additive, such as a dry strength resins and sizing agents. Relating to their function, both of the distributions of them within a paper sheet and a fiber wall should be very important. The depth profile of polyacrylamide (PAM) dry strength resin within a fiber wall was examined by using a technique of an attenuated total reflection/Fourier transform infrared (ATR/FT-IR) analysis combined with gradual etching in previous studies $[1,2]$, and further the effect of the z-directional distribution of a cationic PAM within a paper sheet on the strength of the papers was investigated by the authors [3]. On the other hand an in- plane distribution within a paper sheet was investigated as the distribution on the surface of fibers and around them in the previous report [4]. That is to say, a microscopic ATR/FT-IR imaging analysis was applied to the papers containing a cationic PAM dry strength resin. As a result, a local uneven distribution of the PAM was observed on the fiber surface in part. However, the analytical depth of the ATR/FT-IR is around $1 \mu \mathrm{m}$. The distribution or location of the PAM on the exact fiber surface should be accordingly determined by another method.

In this study, imaging with the latest electron spectroscopy for chemical analysis (ESCA) was adapted to the paper containing the cationic PAM in order to quantify PAM content on the exact fiber surface and further to clarify the distribution or location of the PAM on the exact fiber surface, because the analytical depth of ESCA is up to $10 \mathrm{~nm}$ [5]. The information of the location of PAM could be very important to understand the adsorption behavior of the PAM onto fibers from the view point of the wet-end chemistry, as well as the mechanism of development of paper strength by the addition of PAM dry strength resin.

\section{Experimental}

\subsection{Materials}

Laboratory handsheets made from unbeaten (CSF $662 \mathrm{ml}$ ) and beaten (CSF $155 \mathrm{ml}$ ) fully bleached hardwood kraft pulps containing various amount of a cationic PAM were prepared by an internal application method as described before [1,2]. The method was to make sure of giving as a whole a homogenous PAM distribution in the z-direction as well as $\mathrm{x}-\mathrm{y}$ directions of the paper sheet, and thus an analytical imaging obtained on the paper surface should be the same as that within the paper sheet [2].

The PAM was a commercially available standard cationic PAM from Arakawa Chemical Industries, Ltd (relative molecular mass ; $2.0 \times 10^{6}$, charge density ; 0.6 
$\mathrm{meq} / \mathrm{g}$ ). The total PAM content retained in the paper sheet was determined by the nitrogen analysis and was given as percent on mass of pulp fibers [1,2]. In this study the papers containing PAM at high level content were exclusively employed, since the distribution must be more clearly displayed. Thus the total PAM content was actually $0.8 \%$ for both of the handsheets from unbeaten and beaten pulps.

\subsection{Apparatus}

A scanning ESCA microprobe (Quantera $\mathrm{SXM}^{\mathrm{TM}}$, ULVAC-PHI, Inc., Kanagawa, Japan) was employed on the paper sample. An AlK $\alpha$ peak of $1486.6 \mathrm{eV}$ was used at $15 \mathrm{kV}$ and $1.0 \mathrm{~W}$ as an X-ray source. The beam diameter was $9 \mu \mathrm{m}$. Take-off angle of the photoelectron was $45^{\circ}$. The analyzed area was $250 \mu \mathrm{m} \times 250 \mu \mathrm{m}$ square and the resolution was $173 \times 173$ pixels. The other measuring conditions were as follows: pressure in sample chamber and analyzer, $7.0 \times 10^{-8} \mathrm{~Pa}$; pass energy, $280 \mathrm{eV}$; energy step, $0.8 \mathrm{eV}$; time/step, $1 \mathrm{~ms}$; sweeps, 64 times. The content of nitrogen atom was calculated as a relative mass percent based on a total mass of carbon, oxygen and nitrogen atoms.

\section{Results and discussion}

Figure 1a is a scanning X-ray image (SXI) of the surface of the paper made from the unbeaten pulp containing the PAM, which was an image based on secondary electron measured with this apparatus in order to set a position of the observation. Although the image was a sort of scanning electron microscopic (SEM) image with less resolution than an ordinary SEM, unevenness of the paper surface structure (e.g. surface roughness) appeared to be a black and white zone in this image.

ESCA signal is well known to be largely affected by unevenness of sample surface [5]. Actually, if the unevenness little affects on the signal, $\mathrm{C} 1 \mathrm{~s}$ images of papers may be uniform, since the wood fibers exist all over the analyzing area. However, the obtained $\mathrm{C} 1 \mathrm{~s}$ image (Fig. 1b) which was at the same place as Fig. 1a was not uniform and seemed to reflect the surface unevenness of the paper and fibers. In this image, the relatively stronger signals appeared as yellow color zones and the color gradually shifted to red and black with decreasing in the intensity of the signals. The yellow zone in Fig. 1b, in fact, roughly corresponded to the white zone in Fig. 1a.

Further, O1s image at the same place is shown in Fig. 1c. This image was quite similar to the $\mathrm{C} 1 \mathrm{~s}$ image, namely the zones colored in yellow and red appeared at

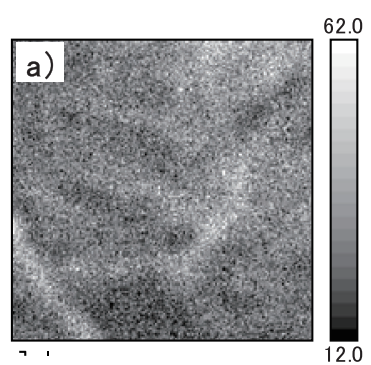

$20 \mu \mathrm{m}$

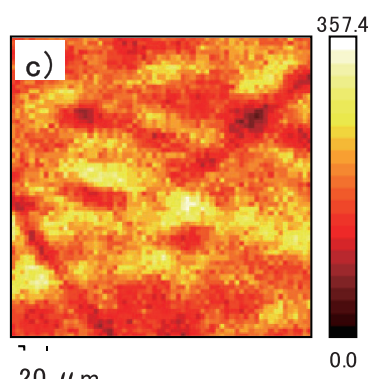

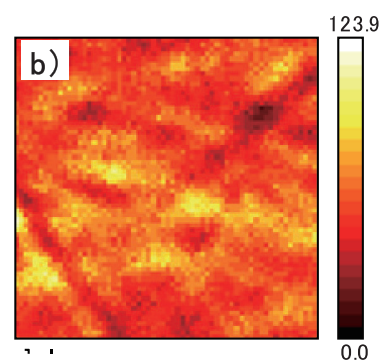

$20 \mu \mathrm{m}$

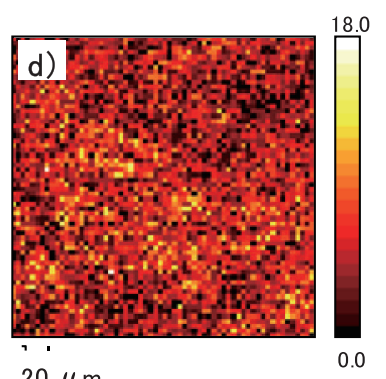

$20 \mu \mathrm{m}$
Fig. 1 Images measured with ESCA of paper from unbeaten pulp containing a cationic PAM (the total PAM content: $0.8 \%$ ) by an internal application method : a) SXI, b) C1s, c) O1s, d) N1s.

the same place. Therefore the signal change from strong to weak seemed to arise from the same unevenness, indicating that the $\mathrm{C} 1 \mathrm{~s}$ and $\mathrm{O} 1 \mathrm{~s}$ signals would be derived from cellulosic fibers in the surface of the paper.

If the PAM was located covering all over the surface of fibers like a film, the N1s image derived from the PAM should be the same as the $\mathrm{C} 1 \mathrm{~s}$ and O1s images. And further the calculated thickness of the film of PAM may be about $13 \mathrm{~nm}$, assuming that the total PAM content was $0.8 \%$, PAM and cellulose had the same density, further fiber wall thickness was about $2 \mu \mathrm{m}$ [6] and a diameter of fiber was about $10 \mu \mathrm{m}$. However, the observed N1s image (Fig. 1d) was far from the above expectation. Hence, the most of PAM should locate within a fiber wall and be distributed near the fiber surface as previously shown in the depth profile within a fiber wall [1,2].

The N1s image (Fig. 1d) also showed that the yellow colored spots were isolated and scattered in the background colored in red, partly in black due to weakness of the N1s signal. The yellow colored spots in Fig. 1d existed around the place colored in yellow in Fig. $1 \mathrm{~b}$ and $1 \mathrm{c}$, at which the strong $\mathrm{C} 1 \mathrm{~s}$ and $\mathrm{O} 1 \mathrm{~s}$ signal were detected. That is to say, the PAM existed on the fiber surface not forming a layer and further a sporadic patch-work pattern distribution was postulated from the following discussion.

The idea of isolated and scattered surface distribution of cationic additives has been first proposed from a study on rates of flocculation of latex particles by 
cationic polymers [7]. The isolated location of the cationic PAM on fiber surface could expect to partly occupy fiber bonding area as a fiber bonding domain in molecular level. And thereby the location could cause an increase of bonding strength per fiber bonded area, which was concluded from difference in optical reflectance during the straining between papers strengthened by beating and addition of PAM [8].

The above descriptions on SXI, C1s, O1s and N1s images for the paper made from the unbeaten pulp were quite similar to those for the paper from beaten pulp, and thus the images for the latter were not shown in this study. This result suggested that beating of fibers little affected on the basic adsorption behavior of the cationic PAM and the resulting distribution pattern on the exact fiber surface. However, beating caused a decrease in yellow colored spots at the N1s image. Furthermore, the nitrogen content from N1s signal was quantitatively analyzed to be 1.5 and $0.9 \%$ for papers from unbeaten and beaten pulps, respectively, even though the total PAM contents was the same for these papers. Therefore, beating should cause the PAM to be less distributed on exact fiber surface.

The measurement with ESCA gave an exact surface PAM content as $7.9 \%$ for the paper made from unbeaten pulp and $4.7 \%$ for that from beaten pulp in spite of the same total PAM content. The both of the exact surface PAM contents were significantly higher than the total PAM content $(0.8 \%)$. This result suggested that the PAM added to the pulp slurry by the internal application method would not be homogeneously distributed within a fiber wall but be comparatively concentrated nearby the surface of fibers. Actually the distribution obtained by the ATR/FT-IR analysis combined with gradual spatter etching supported this explanation $[1,2]$.

These results on the imaging with ESCA can be interpreted as follows: the PAM molecule in the paper stock adsorbed on negatively charged spots at the surface of fibers and fibrils projected from the fibers, leading to aggregate the fibrils [9] and the following collapse of the fibrils to the fiber surface during drying caused the sporadic patch-work surface distribution of the PAM. According to this interpretation, the PAM would be distributed within a fiber wall, even though it mainly located near the fiber surface.

In our previous study, a microscopic ATR/FT-IR imaging of paper surface has shown a generally flat and homogeneous distribution of PAM on the fibers although partly shown a locally uneven distribution [4]. The reason why there were differences between previous and present results would arise from a difference in their analytical depth ; about $1 \mu \mathrm{m}$ for the former and $10 \mathrm{~nm}$ for the latter. The imaging with a microscopic ATR/FT-IR should give rather an in-plane distribution of PAM within a paper or a fiber wall, while the imaging with ESCA should give information on more superficial region.

\section{Acknowledgements}

The authors are grateful to ULVAC-PHI, Inc. for the use of Quantera $\mathrm{SXM}^{\mathrm{TM}}$ and to PFU Co., Ltd. for the financial support.

\section{References}

1. T. Sakaemura, and T. Yamauchi, Japan Tappi J., 63 (11) 1349 (2009).

2. I. Mihara, T. Sakaemura, and T. Yamauchi, Nordic Pulp Paper Res. J., 23 (4) 382 (2008).

3. T. Sakaemura, and T. Yamauchi, Proceedings of the $14^{\text {th }}$ Fundamental Research Symposium held at Oxford, Fundamental Research Communications p.5-8 (2009).

4. T. Sakaemura, I. Mihara, and T. Yamauchi, Senni Gakkaishi, 65 (9) 252 (2009).

5. J. Preston, Proceedings of the 14th Fundamental Research Symposium held at Oxford, p.749 (2009).

6. R. Seth, H. Jang, B. Chan, and C. Wu, Proceedings of the 11th Fundamental Research Symposium held at Cambridge, p.473 (1997).

7. J. Gregory, J. Coll. Inter. Sci., 42, 448 (1973).

8. T. Yamauchi, and T. Hatanaka, Appita J., 55 (3) 240 (2002).

9. P. Myllytie, S. Holappa, J. Paltakari, and J. Laine, Nordic Pulp Paper Res. J., 24 (2) 125 (2009). 